East African Medical Journal Vol. 80. No. 8 August 2003

SERUM ALBUMIN, CREATININE, URIC ACID AND HYPERTENSIVE DISORDERS OF PREGNANCY

B. L. Salako, MBBS, FWACP, Department of Medicine, A. Odukogbe, MBBS, FWACS, FMCOG, O. Olayemi, MBBS, FWACS, K. S. Adedapo, MSc, FWACP (Lab Med) Department of Chemical Pathology, University College Hospital, C.O. Aimakhu, MBBS, FWACS, F.E. Alu, MBBS, FWACS, Department of Obstetrics and Gynaecology, University College Hospital, Ibadan Nigeria, and B. Ola, MBBS, FWACS, Birmingham Women Hospital, Birmingham, England

Request for reprints to: Dr. B.L Salako, Department of Medicine, University College Hospital, Ibadan, PMB 5116, Ibadan, Nigeria

\title{
SERUM ALBUMIN, CREATININE, URIC ACID AND HYPERTENSIVE DISORDERS OF PREGNANCY
}

\author{
B. L. SALAKO, A.T.A. ODUKOGBE, O. OLAYEMI, K.S. ADEDAPO, C.O. AIMAKHU, F.E. ALU and B. OLA
}

\begin{abstract}
Background: Pre-eclampsia is a form of hypertensive disorder of pregnancy. It is a common cause of both maternal and perinatal morbidity and mortality in both developed and developing countries.

Objective: To evaluate the possibility of early prediction of hypertensive disorders of pregnancy using single estimation of serum protein, creatinine and uric in serum samples of healthy primigravidae with singleton pregnancy.

Setting: University College Hospital, Ibadan.

Subjects: Fifty nine normortensive primigravidae.

Methods: Fifty nine healthy normotensive primigravidae with singleton pregnancy who booked for antenal care and delivered at the University College Hospital, Ibadan had single estimations of their serum albumin, creatinine and uric acid levels at booking before the 20th week of pregnancy. The women were followed up longitudinally throughout pregnancy. Results: Pre-eclampsia occurred in five of the patients $(21.7 \%)$, two had pregnancy induced hypertension only $(8.7 \%)$ while 16 remained normotensive $(69.6 \%)$. The difference in the mean serum concentration of uric acid $(0.162 \pm 0.02 \mathrm{mmol} / \mathrm{L})$ and creatinine $(93.70 \pm 10.08$ $\mu \mathrm{mol} / \mathrm{L})$ respectively were not statistically significant $(\mathbf{p}>0.05)$. However, the difference in the mean serum albumin levels $(4.06 \pm 0.06$ versus $3.71 \pm 0.33 \mathrm{gm} / \mathrm{dl})$ was significantly higher in the pre-eclampsia group $(\mathbf{p}<\mathbf{0 . 0 5})$. The predictive performance of these tests was generally low whether alone or in combination.

Conclusion: Single estimation of serum uric acid and creatinine levels early in pregnancy are of little value in the prediction of pre-eclampsia. A large study is recommended to properly define the value of serum albumin levels in pregnancy in the prediction of pre-eclampsia in the light of the findings of this study.
\end{abstract}

\section{INTRODUCTION}

Pre-eclampsia is a form of hypertensive disorder of pregnancy. Its general prevalence is between $2-8 \%$ of pregnancies (1). In Ibadan the prevalence is about $9.7 \%$, which is a little higher than the general prevalence (2). It is a common cause of both maternal and perinatal morbidity and mortality in both developed and developing countries (3). Low dose aspirin and calcium supplementation have been tried in the prevention of the disease but their use is not without risks $(4,5)$. Indeed, several clinical, biophysical and biochemical screening tests have been proposed. Some of these tests include the use of the angiotensin II pressor response (6), the roll-over test (7), and the isometric handgrip exercise test (8), and mean arterial pressure (9). Most of these studies had limitations as screening tests in the clinical setting because of their complexity and high incidence of false positive results.

Many biochemical markers of pre-eclampsia have been recognised in maternal serum. These include uric acid, creatinine and albumin among others. The results of some studies in normotensive pregnant women suggest that serum uric acid levels begin to rise before the appearance of hypertension and proteinuria (10). Plasma concentrations of creatinine in preeclampsia have been shown to be significantly higher than in the normotensive subjects (11). However, plasma albumin decreases in pregnancy and is reduced further in pregnancies complicated by pre-eclampsia(12). Most of the studies involving estimations of serum uric, albumin and creatinine levels as biochemical markers for the prediction of the subsequent development of pre-eclampsia, were performed late in the second and third trimesters of pregnancy when the disease process was usually manifest(10-12). An ideal predictive test for pre-eclampsia should be easy to perform early in the pregnancy, simple, reproducible and of high positive predictive value. At present no one test fulfils all these criteria (13). It is important therefore to identify women who are at high risk of developing the disease early in pregnancy. This is because early identification of biochemical markers of the disease 
would not only facilitate selective recruiting of those at increased risk for pre-eclampsia but also help in determining those patients who were more likely to benefit from interventional measures should a therapeutic intervention prove successful.

This study therefore involves single estimations of serum albumin, creatinine and uric acid levels of healthy normotensive primigravidae who booked for antenatal care at the University College hospital (UCH), Ibadan before the 20th week of pregnancy.

\section{MATERIALS AND METHODS}

This study was done at the antenatal clinic of the University College Hospital, Ibadan, Nigeria within a period of 12 months. All consenting healthy primigravidae attending the antenatal clinic of the University College Hospital (UCH) Ibadan within the study period were recruited for the study if they were first seen before 20th week of pregnancy and they were excluded from the study if they were first seen after the 20th week of pregnancy, had multipurpose or multiple gestation. Subjects with history of chronic hypertension, diabetes mellitus, renal disease or collagen vascular disease were also excluded. Women who at entry into the study had a blood pressure that was greater than or equal to $140 / 90 \mathrm{mmHg}$, or had proteinuria by the dipstick measurement greater than $300 \mathrm{mg} / \mathrm{L}(1+)$ were excluded. Those who met the inclusion criteria were followed up at each prenatal clinic till delivery. Subjects were further excluded from the final analysis if they failed to make at least two routine prenatal clinic visits or did not deliver in UCH Ibadan after being recruited. The joint Ethical Committee of the University of Ibadan/University College Hospital, Ibadan, approved the study protocol. All the eligible patients were adequately counseled and their informed consent obtained before they were included in the study. At the time of booking a complete clinical history was taken from each subject with emphasis on her age, date of the last menstrual period, family and social history and history of any medical condition such as hypertension, diabetes mellitus or renal disease and drug use. This was followed by a complete physical examination and an assessment of the lower extremity for oedema. The weight and height of the subjects were measured and the blood pressure taken twice on the right arm in the sitting position with the patient at rest using a mercury sphygmomanometer with an appropriate cuff placed at heart level. The systolic blood pressure (SBP) was first estimated by palpation of the radial artery pulsation following inflation of the cuff. The cuff was then rapidly deflated. It was then re-inflated to about $30 \mathrm{mmHg}$ above the estimated SBP and then gradually deflated while listening with a stethoscope over the cubital fossa. Systolic blood pressure and diastolic blood pressure(DBP) were respectively taken as the level of pressure at which Korotkoff sounds were clearly heard for the first time(Phase I) and the level at which they became muffled (Phase IV). The mean values were used in the analysis. The mean arterial pressure (MAP) was calculated as DBP $+(\mathrm{SBP}-\mathrm{DBP}) / 3 \mathrm{mmHg}$. Hypertension was taken as blood pressure greater than or equal to $140 / 90 \mathrm{mmHg}$.

A dipstick urine measurement for protein was performed on a random urine specimen collected by the patient. Testing was performed with multistix reagent strips (Bayer) and results were reported as negative, trace, $1+, 2+, 3+$ or $4+$. All those who met the study criteria had a single estimation of their serum albumin, creatinine and uric acid levels at booking. Analysis of the serum samples was performed at the chemical Pathology laboratory of the $\mathrm{UCH}$, Ibadan using appropriate biochemical assay methods. The samples were analysed the same day, serum albumin was estimated by the dye binding method using Bromocresol Green (BCG) while serum creatinine was determined calorimetically by the Jaffe reaction of Bonsnes and Taussky. Serum uric acid was estimated by the modified alkaline phosphotung state method with sodium carbonate as alkali.

The patients then received routine prenatal care and at each clinic visit the blood pressure was measured and the urine tested for protein. Urine culture and sensitivity tests were performed when necessary to exclude urinary tract infection as a cause of any observed proteinuria. All data obtained were coded and keyed into a computer running the EPI-INFO version 6 programme for analysis. Statistical analyses for categorical variables were based on the Chi square tests whereas continous variables were based on the Kruskal-Wallis test and analysis of variance (ANOVA) for repeated measurements. Results were expressed as means $+\mathrm{SD}$ except where otherwise stated. A P-vaule of $<0.05$ was considered statistically significant (providing a $95 \%$ confidence interval).

The predictive performance of the individual tests was estimated by an arbitrary selection of a cut-off point (threshold value) for each parameter. Threshold values of $\geq 0.21 \mathrm{~mm} 01 / \mathrm{L}$ for uric acid, $\geq 88.4 \mathrm{um} 01 / \mathrm{L}$ for creatinine and $\geq 4 \mathrm{gm} / \mathrm{dl}$ for albumin were chosen as predictive for the development of preeclampsia. The predictive performance of serum uric acid and creatinine in combination was also assessed using a uric acid/ creatinine ration of $\geq 0.002$.

\section{RESULTS}

A total of 59 primigravidae with singleton pregnancy were recruited before their 20th week of pregnancy. Two of them had abortions, 13 did not make the mandatory subsequent prenatal clinic visits, and 21 did not deliver in $\mathrm{UCH}$, Ibadan due to industrial actions. Of the 23 primmigravidae who completed the study five $(21.7 \%)$ had pre-eclampsia, two $(8.7 \%)$ had pregnancy induced hypertension only while $16(69.6 \%)$ remained normotensive. One of those who developed pre-eclampsia later had intrapartum eclampsia and died. Oedema and proteinuria were mild in all the preeclampsia cases except in the only eclampsia case where there was marked oedema and proteinuria.

Table 1 shows the maternal characteristics of the study groups. The mean maternal age, gestational age, systolic and diastolic blood pressures and mean arterial blood pressure (MAP) of the pre-eclampsia group at booking did not significantly differ from those of the normotensive group. However the differences in the mean systolic and diastolic blood pressures and mean arterial blood pressure of the two groups at delivery were statistically significant $(\mathrm{p}<0.05)$.

Table 2 shows the mean serum concentrations for albumin, creatinine and uric acid in the pre-eclampsia and normotensive groups. There was no significant difference in the mean values of uric acid and creatinine in the two groups. The difference in the mean serum albumin concentration in the two groups was however statistically significant $(\mathrm{p}<0.05)$ with higher values in the pre-eclampsia group.

Table 3 shows the predictive values of the serum 
concentrations of the parameters tested. None of the five patients in whom pre-eclampsia developed had a serum uric acid value $\geq 0.21 \mathrm{mmoI} / \mathrm{L}$; one had serum albumin value $\leq 4 \mathrm{gm} / \mathrm{dl}$; four had serum creatinine values $\geq 88.4 \mathrm{umoI} / \mathrm{L}$ while four had uric acid/creatinine ratio $\geq 0.002$.
Three patients in whom pre-eclampsia did not develop had serum uric acid values $>0.21 \mathrm{mmoI} / \mathrm{L} ; 14$ had serum albumin values $\leq 4 \mathrm{gm} / \mathrm{dl}$; 11 had serum creatinine values $\geq 88.4 \mathrm{umoI} / \mathrm{L}$ while $12 \mathrm{had}$ uric acid/creatinine ratio $>0.002$.

\section{Table 1}

Maternal characteristics of study groups

\begin{tabular}{llll}
\hline & $\begin{array}{l}\text { Normotensive } \\
(\mathrm{n}=16)\end{array}$ & $\begin{array}{l}\text { Pre-eclampsia } \\
(\mathrm{n}=5)\end{array}$ & $\begin{array}{l}\text { Significance } \\
(\mathrm{p} \text {-value })\end{array}$ \\
\hline Mean maternal age (years) & $27.0 \pm 3.71$ & $28.8 \pm 2.97$ & 0.280 \\
Mean gestational age at booking (weeks) & $15.5 \pm 3.05$ & $14.3 \pm 3.09$ & 0.231 \\
Mean systolic blood pressure at booking (mmHg) & $114.0 \pm 10.4$ & $117 \pm 20.7$ & 0.781 \\
Mean diastolic blood pressure at booking (mmHg) & $68.0 \pm 8.45$ & $70.0 \pm 8.94$ & 0.521 \\
Mean arterial blood pressure at booking (mmHg) & $83.0 \pm 8.81$ & $85.0 \pm 12.0$ & 0.787 \\
Mean gestational age at delivery (weeks) & $38.0 \pm 3.54$ & $36.6 \pm 3.16$ & 0.240 \\
Mean systolic blood pressure at delivery (mmH) & $120.0 \pm 7.07$ & $146.0 \pm 25.7$ & $0.044^{*}$ \\
Mean diastolic blood pressure at delivery $(\mathrm{mmHg})$ & $68.0 \pm 4.47$ & $91.0 \pm 17.7$ & $0.036^{*}$ \\
Mean arterial blood pressure at at delivery(mmg) & $85.0 \pm 3.91$ & $109.0 \pm 20.2$ & $0.022^{*}$ \\
\hline
\end{tabular}

- Values are mean \pm SD

$* \mathrm{P}<0.05$ significant

Table2

Mean serum values for albumin, creatinine and uric acid at booking

\begin{tabular}{llll}
\hline & $\begin{array}{l}\text { Normotensive } \\
(\mathrm{n}=16)\end{array}$ & $\begin{array}{l}\text { Pre- } \\
\text { eclampsia } \\
(\mathrm{n}=5)\end{array}$ & P-value \\
\hline Serum Albumin $(\mathrm{gm} / \mathrm{dl})$ & $3.71+0.33$ & $4.06+0.60$ & $0.042^{*}$ \\
Serum Creatinine $(\mu \mathrm{mol} / \mathrm{L})$ & $90.61+18.96$ & $93.70+10.08$ & 0.736 \\
Serum Uric Acid $(\mathrm{mmol} / \mathrm{L})$ & $0.162+0.05$ & $0.162+0.02$ & 0.482 \\
\hline
\end{tabular}

- Values are mean $\pm \mathrm{SD}$

$* \mathrm{P}<0.05$ significant

Table 3

Predictive values of serum albumin, creatinine and uric acid

\begin{tabular}{lllll}
\hline & Albumin & Creatinine & $\begin{array}{l}\text { Uric } \\
\text { Acid }\end{array}$ & $\begin{array}{l}\text { Uric Acid/ } \\
\text { Creatinine } \\
\text { Ratio }\end{array}$ \\
\hline Pre-eclampsia (n=5) & & & 0 & 4 \\
Normotensive (n=16) & 14 & 4 & 3 & 12 \\
Sensitivity (\%) & 20 & 11 & 0 & 80 \\
Specificity (\%) & 12.5 & 80 & 81 & 25 \\
Positive Predictive Value (\%) & 6.7 & 26.7 & 0 & 25 \\
Negative Predictive Value (\%) & 33 & 83 & 72 & 80 \\
\hline
\end{tabular}

Predictive levels- Albumin $\leq 4 \mathrm{gm} / \mathrm{dl}$

Creatinine $\geq 88.4 \mu \mathrm{mol} / 1$

Uric acid $>0.21 \mathrm{mmol} / \mathrm{L}$

Uric acid/creatinine ratio $\geq 0.002$ 
This gives serum uric acid a sensitivity of $0 \%$, a specificity of $81 \%$, a positive predictive value of $0 \%$ and a negative predictive value of $72 \%$. Serum creatinine and the uric acid/creatinine threshold ratio had the highest sensitivity of $80 \%$ each.

The specificity was however low for all the other parameters (range 12.5-31\%). The positive predictive values for all the parameters were also generally low with serum creatinine having the highest $26.7 \%$ ) and serum uric acid the lowest $(0 \%)$. The negative predictive values ranged from $33 \%$ for serum albumin to $83 \%$ for serum creatinine. On the whole, the performance of serum creatinine appeared better than those of the other parameters and when serum uric acid was combined with serum creatinine the sentisitivity was high $(80 \%)$, the specificity low $(25 \%)$ with a low positive predictive value $(25 \%)$ and a high negative predictive value $(80 \%)$.

\section{DISCUSSION}

This study was designed to prospectively evaluate the possibility of early prediction of the subsequent development of pre-eclampsia using single estimation of levels of some known biochemical substances affected by the disease in serum samples of healthy primigravidae with singleton pregnancy. The selection of primigravidae with singleton pregnancy was based on the knowledge that these groups of women are more prone to developing the disease when all other risk factors are excluded. Those with conditions that could place them at increased risk of the disease such as multiple gestation, chronic hypertension, diabetes mellitus and renal disease were carefully excluded from the study.

For a predictive test to be of value in the identification of women at high risk of developing pre-eclampsia, the test should be altered early enough in pregnancy to allow for the institution of preventive measures (14). This was the justification for the selection of the subjects before the 20th week of pregnancy for the study. In our center however, the monthly booking and delivery rates are about 300 and 150 respectively: in this figures only about $30 \%$ book earlier than 20 weeks. This with the strict criteria used for subjects' selection explains the relatively low sample size.

The concomitant study of several tests was to compare their respective performances alone and in combination. The mean systolic and diastolic blood pressure and the mean arterial pressure values at delivery were all significantly higher in the pre-eclampsia group. This was expected in view of the criteria used for the diagnosis of the disease. The mean arterial pressure (MAP) has been shown to be predictive of pre-eclampsia, although some other studies indicated otherwise (9).

There was no significant difference in the mean values of uric acid and creatinine in the two groups. This was contrary to the findings of other workers that serum uric acid and creatinine levels are usually raised in patients destined to develop pre-eclampsia $(10,11)$.
However, the inclusion criteria used in some of these studies were slightly different from those of the present study. For instance, some of the studies included patients with mixed parity (nulliparous and multiparous women), some with chronic hypertension and renal disease $(10,15)$. In addition the tests were carried out late in pregnancy when the effects of the disease were often manifest with patients already having symptoms. It was also possible that the effect of pre-eclampsia on the renal system early in pregnancy might be minimal as to produce any detectable change in the serum levels of these substances. Hayashi et al demonstrated that abnormally high blood creatinine levels are seldom observed and those of uric acid often are normal even though the clearance was reduced in patienst with preeclampsia (16). It was interesting to find that the mean serum albumin concetration in those remained normotensive was lower than that of the pre-eclampsia group, although the values were within the normal range. This was at variance with the generally known concept of hypoalbuminaemia being a feature of pregnancies complicated by pre-eclampsia (12). However, significant proteinuria was not a feature of the patients at booking when the estimations were made, therefore, a larger study is required to properly define the value of serum albumin estimation early in pregnancy in the prediction of pre-eclampsia.

The sensitivity, specificity and predictive values of the various parameters in this study were consistent with those of other studies screening a low risk population (13). A screening test for pre-eclampsia in allow risk or general obstetric population should be able to identify most women who ultimately develop the disease. In other words, it must have a high sensitivity and a high positive predictive value with an acceptable false positive rate. None of the parameters studied here fulfilled these requirements.

There were a number of limitations in the conduct of this study. First, recruitment of patients into the study using the strict exclusion criteria affected the number eligible for the study because our patients in this part of the world rarely book early in pregnancy unless they develop complications. Secondly, the incessant industrial actions by the health workers in the hospital where the study was conducted resulted in most of the patients earlier recruited into the study delivering outside the University College Hospital, Ibadan.

In conclusion, the findings of this study showed that single estimations of serum uric acid and creatinine concentrations early in pregnancy are of little value in the prediction of subsequent development of preeclampsia, either alone or in combination. However, mean serum albumin level was significantly higher in those who developed pre-eclampsia. A larger study is therefore recommended to properly define the value of estimation of serum albumin levels in early pregnancy in the prediction of pre-eclampsia. 


\section{REFERENCES}

1. Duley L. The management of pre-eclampsia. Obstet gynaecol. 2000; 2:45-48.

2. Salako B.L., Olayemi D., Odukogbe A. et al. Microalbuminuria in pregnancy as a predictor of preeclampsia and eclampsia (in press). (WAJM).

3. Robson S.C. Hypertension and renal disease in pregnancy. In: Dewhurst's Textbook of Obstetrics and Gynaecology for Postgraduates. 6th Ed; 1999 (Edmonds D. K, ed). Blackwell Scientific Publications Ltd, London. pp 166-185.

4. McParland P.M., Pearce J.M., and Chamberlain G.V.P. Doppler ultrasound and aspirin in recognition and prevention of pregnancy induced hypertension. Lancet 1990; 335:15521555.

5. Belizan J.M., Villar J., Gonzalez L. et al. Calcium supplementation to prevent hypertensive disorders of pregnancy. New Eng. J. Med. 1991; 325: 1399-1403.

6. Morris J.A., O'Grady J.P., Hamilton C.J., and Davidson E.C. Vascular reactivity to angiotensin II infusion during gestation. Am. J. Obstet. Gynaecol. 1978; 130:379-382.

7. Gant N.F., Chand S., Worley R.J., et al. A clinical test useful for predicting the development of acute hypertension in pregnancy. Am. J. Obstet. Gynaecol. 1974; 120: 1-7.

8. Degani S., Abinader E.; Eibschitz I., et al. Isometric exercise test for predicting gestational hypertension. Obstet. Gynaecol. 1985; 65:652-654.
9. Redman C.W.G., Beilin L.J.,Bonnar J., and Wilkinson R.H. Plasma urate measurements in predicting fetal death in hypertensive pregnancy. Lancet 1976; i: 1370-1373.

10. Fay R.A., Bromhan D.R., Books J.A. and Gebski V.J. Platelets and uric acid in the prediction of pre-eclampsia. Am. J. Obstet. Gynaecol. 1985; 152:1038-1039.

11. Wakwe V.C. and Abudu O.O. Estimation of plasma uric acid in pregnancy induced hypertension: Is the test still relevant? Afr. J. Med. Sci. 1999; 28:155-158.

12. McCartney C.P.; Schumacher G.F.B.; and Spargo B.H. Serum Proteins in patients with toxaemic goomerular lesions. Am. J. Obstet. Gynaecol. 1971; 580-590.

13. Conde-Agudelo A,, Lede R., and Belizan J. Evaluation of methods used in the prediction of hypertensive disorders of pregnancy. Obstet. Gynaecol. Surv. 1994; 49:210-222.

14. Eskenazi B., Fenster L., and Sidney S.A. Multivariate analysis of risk factors for pre-eclampsia. JAMA 1991; 266: 237-241.

15. Conde -Angudelo A.; Belizan J.M., Lede R., and Bergel E.F. What does an elevated mean arterial pressure in the second half of pregnancy predict-Gestational hypertension or pre-eclampsia? Am. J. Obstet. Gynaecol. 1993; 169:509514.

16. Hayashi T. and Philadelphia P. Uric acid and endogenous creatinine clearance studies in normal pregnancy and toxaemias of pregnancy. Am. J. Obstet. Gynaecol. 1956; 71:859-870

We would like to carry out the following corrections in our April 2003 issue, Volume 80 Number 4.

- A.J. Kinsara Pages 200-203,

Abstract: in the conclusion, line 2 should read as; it is NOT needed routinely.

- K.O. Amisu, A.O. Coker, S.L.W. on and R.D. Isokpehi, Pages 218-222, In the title, the word "Butzlieri" should read "Butzleri"

We sincerely apologise for these errors. 